\title{
ANÁLISIS TECNO-ECONÓMICO DEL DESPLIEGUE DE UNA ARQUITECTURA DE RED VIRTUALIZADA
}

\author{
Jesús Calle-Cancho ${ }^{1}$, Javier Carmona-Murillo², David Cortés-Polo ${ }^{1}$ \\ y José-Luis González-Sánchez ${ }^{1}$ \\ ${ }^{1}$ Centro Extremeño de Investigación, Innovación Tecnológica y Supercomputación (CénitS), Cáceres, España \\ ${ }^{2}$ Departamento de Ingeniería de Sistemas Informáticos y Telemáticos, Universidad de Extremadura, Cáceres, España
}

\begin{abstract}
RESUMEN
En los últimos años, el incremento exponencial del tráfico de datos móviles unido al despliegue de nuevos servicios sobre las redes actuales ha propiciado que los operadores de red busquen nuevas tecnologías para adaptarse, entre las que destacan dos principalmente: Redes Definidas por Software (SDN) y la Virtualización de Funciones de Red (NFV). Estas tecnologías aportan dinamismo a las redes de próxima generación. Dado que la migración a estas nuevas tecnologías conlleva un coste elevado para los operadores, éstos buscan soluciones que ofrezcan un aumento de las capacidades de la red, reduciendo los costes de infraestructura (CAPEX) y operación (OPEX). En este trabajo se desarrolla un análisis y evaluación de costes que serían necesarios para que una red real se convierta en el principal operador de Extremadura.
\end{abstract}

\section{PALABRAS CLAVE}

Redes Definidas por Software, Virtualización de Funciones de Red, EPC, OPEX, CAPEX

\section{INTRODUCCIÓN}

Debido al continuo desarrollo de las redes de comunicaciones móviles en los últimos años, la realización de una gestión eficiente de la red se ha convertido en uno de los mayores desafíos en entornos de red de próxima generación. Además, la aparición de multitud de servicios heterogéneos ofertados por los operadores, han impulsado la creación de nuevos mecanismos y arquitecturas de gestión de redes, capaces de soportar los nuevos servicios emergentes. Estos avances han generado un crecimiento exponencial del tráfico global de datos móviles estimándose un consumo mensual de 77,5 exabytes en 2022 (Cisco 2019).

Por otro lado, todos estos cambios están teniendo un gran impacto en las estrategias económicas llevadas a cabo por los operadores de red. El aumento de los requisitos de eficiencia y disponibilidad por parte de los usuarios va ligado a un incremento de los costes de todo ello. Por lo tanto, las decisiones de planificación y diseño de las redes deben tener en cuenta las estimaciones de costes con la mayor precisión posible. Para ello se utilizan modelos basados en CAPEX y OPEX (S. Verbrugge et al. 2006) (B. Naudts et al. 2016).

En definitiva, los operadores de red requieren de nuevos mecanismos para cubrir de manera eficiente (técnica y económicamente) las necesidades que han ido apareciendo en los nuevos entornos de red. Estos mecanismos deben ser capaces de reservar dinámicamente los recursos de la red para proporcionar la flexibilidad requerida por los operadores de red (B. Blanco et al. 2017). Por ello, SDN y NFV son vistos como una gran oportunidad para hacer frente a la naturaleza dinámica de las redes de próxima generación.

Este artículo presenta un análisis del despliegue de mecanismos basados en SDN y NFV. Para ello, se ha elaborado un modelo basado en el CAPEX y OPEX, para analizar de manera cuantitativa el despliegue de una arquitectura de red virtualizada sobre una red real en Extremadura (RCT).

El resto del artículo está organizado de la siguiente manera. La sección 2 presenta un estado del arte de SDN y NFV. En la sección 3 se define y presenta el modelo de costes que se utilizará para realizar el análisis del despliegue de una arquitectura de red virtualizada sobre la RCT. La sección 4 muestra los resultados comparativos entre los costes necesarios para el despliegue de una arquitectura de red basada en SDN/NFV y una arquitectura de red tradicional, así como los costes asociados al despliegue del EPC (Evolved Packet Core) virtualizado. Por último, en la sección 5 se presentan las conclusiones del trabajo. 


\section{ESTADO DEL ARTE: SDN Y NFV}

La tecnología de las Redes Definidas por Software surge como un nuevo paradigma de red, cuya principal característica es la separación del plano de datos del plano de control, con el objetivo de simplificar la gestión y configuración de la red (B.A.A. Nunes et al. 2014). SDN proporciona una vista global de la red a través de un controlador de red centralizado. Por lo tanto, el plano de control queda centralizado en el controlador de la red que, a su vez, gestiona el plano de datos a través de protocolos abiertos como OpenFlow.

Por otro lado, la aparición de la tecnología de NFV ha sido muy importante desde el punto de vista del aprovisionamiento de servicios. Este paradigma tiene como principal objetivo el desacoplar las funciones de red de los dispositivos físicos en los cuales se ejecutan. Además, NFV tiene el potencial de proporcionar reducciones significativas de CAPEX y OPEX, y de facilitar y flexibilizar el despliegue de nuevos servicios con más agilidad (B. Han et al. 2015), permitiendo alcanzar los requisitos de baja latencia y alta fiabilidad requerida por los servicios que se ofrecerán en las futuras redes 5G (J.A. Cabrera et al. 2019). Los paradigmas SDN y NFV están íntimamente relacionados (R. Mijumbi et al, 2016) y, con una integración eficiente de ambos, se puede conseguir un importante ahorro de costes.

La virtualización de red persigue una división de los recursos físicos de una red en segmentos o slices. Cada slice está aislada de las demás y pueden compartir infraestructura física en paralelo. Por ello, se acuñó el término de Cloud-RAN (I-F. Akyildiz et al. 2015) que permite desacoplar el procesamiento de banda base de las propias estaciones base, ofreciendo un despliegue más fácil y flexible, a diferencia de las estaciones base (BS) tradicionales. De esta manera, aparece el concepto de SBS (BS definida por software), en la que un número determinado de $\mathrm{BS}$ virtuales pueden ser desplegadas simultáneamente.

Numerosos estudios establecen que SDN y NFV conducen a una reducción significativa del CAPEX para los operadores de red (B. Naudts et al. 2016) (R. Mijumbi et al. 2016). En relación al OPEX (E. Hernández et al. 2015), los procesos operativos automatizados podrían reducir la intervención humana, reduciendo los costes de personal y las operaciones de red que generen fallos.

\section{MODELO DE COSTES}

En esta sección se presenta el modelo de costes que se utilizará en secciones posteriores, para analizar y evaluar de manera cuantitativa el despliegue de una arquitectura de red virtualizada. El modelo utilizado establece que los costes totales de un determinado operador pueden ser divididos en:

- Costes de capital (CAPEX). Son inversiones de capital que crean beneficios. El CAPEX engloba los costes de la infraestructura fija.

- Costes de operación (OPEX). También llamados costes de funcionamiento u operativos. El OPEX representa el coste necesario para mantener operativa la infraestructura.

Para poder evaluar y comparar convenientemente los costes del despliegue de una arquitectura de red virtualizada, que haga uso de los nuevos paradigmas emergentes, es necesario analizar también los costes a los que los operadores tendrían que hacer frente si se usara una arquitectura de red tradicional.

En realidad, una RAN (Red de Acceso Radio) virtualizada (C-RAN) se trata de una evolución de una RAN tradicional, en la que el elemento principal pasa a ser una BS virtualizada (vBS), es decir, un número determinado de BS virtuales pueden ser desplegadas simultáneamente sobre una BS física (D. Pompili et al. 2015). La estación base que albergue varias vBS la denominaremos SBS.

\subsection{CAPEX y OPEX en una 5 ed de $\$$ cceso 7 radicional}

En esta sección se modelan los costes relacionados con una red de acceso radio tradicional, que permitirán evaluar el impacto relativo al despliegue de la infraestructura de red necesaria y su mantenimiento como si fuera un operador de red tradicional. Se asume que en un área A existen un determinado número de operadores $\left(\mathrm{N}_{\mathrm{op}}\right)$ que se encuentran dando cobertura a un conjunto de clientes. El número de estaciones base para cubrir el área A depende del radio de cobertura de la estación base $\left(\mathrm{R}_{\max }\right)$ y del número de usuarios en $\mathrm{A}$. Por lo tanto, el número total de estaciones base requeridas por cada operador $\left(\mathrm{N}_{\mathrm{BSO}}\right)$ viene dado por la Ec. 1 . 


$$
N_{B S O}=\frac{A}{\pi \cdot R_{\max }^{2}}
$$

Si $\lambda$ refleja la densidad de usuarios por unidad de área y operador, se puede definir el número total de usuarios por operador $\left(\mathrm{N}_{\mathrm{UOp}}\right)$ como se muestra en la Ec. 2.

$$
N_{U O p}=\lambda \cdot A=\lambda \cdot \pi \cdot N_{B S O} \cdot R_{\text {max }}^{2}
$$

Siendo $\mathrm{C}_{\mathrm{dBS}}$ los costes asociados al despliegue inicial de una estación base y $\mathrm{C}_{\mathrm{BS}}$ los costes de cada estación base, se puede definir el CAPEX de una arquitectura RAN tradicional como se muestra en la Ec. 3.

$$
C A P E X_{R A N}=\sum_{i=1}^{N_{o p}} \sum_{j=1}^{N_{B S O_{i}}}\left(C_{d B S}+C_{B S}\right)_{i j}
$$

Por otro lado, la Ec. 4 define el OPEX total de una arquitectura RAN tradicional. Para ello se tendrán en cuenta la energía consumida por BS $\left(\mathrm{P}_{\mathrm{BS}}\right)$, el coste del $\mathrm{kWh}\left(\mathrm{C}_{\mathrm{kWh}}\right)$, los costes asociados a la operación, mantenimiento y administración $\left(\mathrm{C}_{\mathrm{OAM}}\right)$ y los costes de alquiler $\left(\mathrm{C}_{\mathrm{ALQ}}\right)$.

$$
O P E X_{R A N}=\sum_{i=1}^{N_{O p}} \sum_{j=1}^{N_{B S O}}\left(P_{B S} \cdot C_{k W h}+C_{O A M}+C_{A L Q}\right)_{i j}
$$

$\mathrm{P}_{\mathrm{BS}}$ engloba la potencia de todas las antenas que conforman la estación base. Considerando que una estación base está formada por un número de antenas determinado $\left(\mathrm{N}_{\mathrm{a}}\right)$, con una potencia determinada cada una $\left(\mathrm{P}_{\mathrm{a}}\right)$, se puede estimar la potencia eléctrica total de cada BS, tal y como se muestra en la Ec. 5.

$$
P_{B S}=N_{a} \cdot P_{a}
$$

\subsection{CAPEX y OPEX en una 5 ed de $\$$ cceso 9 irtualizada}

A continuación, se modelan los costes relacionados con una red de acceso radio virtualizada. Se asume que en un área A existe un determinado número de estaciones base virtuales vBS, desplegadas sobre SBS, que se encuentran dando cobertura a un conjunto de usuarios. El número de SBS requerido para cubrir el área A estará determinado por el radio de cobertura de la SBS $\left(\mathrm{R}_{\max }\right)$ y por el número total de usuarios en el área $\mathrm{A}$, tal y como se muestra en la Ec. 6.

$$
N_{S B S}=\frac{A}{\pi \cdot R_{\max }^{2}}
$$

Si $\lambda$ refleja la densidad de usuarios por unidad de área y operador; y $\mathrm{N}_{\mathrm{sl}}$ define el número de slices (vBS) por cada SBS, se tiene que el número total de usuarios en un área $\mathrm{A}$ es $\mathrm{N}_{\mathrm{UT}}$ (Ec. 7).

$$
N_{U T}=\lambda \cdot A=\sum_{i=1}^{N_{S l}}\left(\lambda \cdot \pi \cdot N_{S B S} \cdot R_{\text {max }}^{2}\right)
$$

Además, $\mathrm{C}_{\mathrm{SBS}}$ representa el coste de una SBS. Se asume que el coste total de una SBS aumenta linealmente con respecto al número de vBS desplegadas en ella, tal y como se muestra en la Ec. 8.

$$
C_{S B S}=C_{B S} \cdot\left(1+0,2 \cdot\left(N_{s l}-1\right)\right)
$$

Siendo $\mathrm{C}_{\mathrm{dsBS}}$ el coste asociado al despliegue inicial de una SBS, se puede definir el CAPEX total de una arquitectura C-RAN como se muestra en la Ec. 9.

$$
C A P E X_{C-R A N}=C_{d S B S-\text { total }}+C_{S B S-\text { total }}=N_{S B S} \cdot\left(C_{d S B S}+C_{S B S}\right)
$$

Por otro lado, la Ec. 10 define el OPEX para una arquitectura C-RAN. Estará formado por los costes derivados del consumo energético de las SBS, que serán calculados a partir de la potencia de la SBS (PSBS) y del coste del $\mathrm{kWh}\left(\mathrm{C}_{\mathrm{kWh}}\right)$. Además, se tendrán en cuenta costes asociados a la operación, mantenimiento y 
administración $\left(\mathrm{C}_{\mathrm{OAM}}\right)$ y los costes de alquiler $\left(\mathrm{C}_{\mathrm{ALQ}}\right)$. Cada $\mathrm{SBS}$ tiene más capacidades que una $\mathrm{BS}$ tradicional, por lo que $\mathrm{P}_{\mathrm{SBS}}$ dependerá del número de vBS que se desplieguen sobre ella.

$$
O P E X_{C-R A N}=\sum_{i=1}^{N_{S B S}}\left(P_{S B S} \cdot C_{k W h}+C_{O A M}+C_{A L Q}\right)_{i}
$$

Considerando que cada vBS está formada por un número de antenas determinado $\left(\mathrm{N}_{\mathrm{a}}\right)$, se puede estimar que la potencia eléctrica de cada SBS $\left(\mathrm{P}_{\mathrm{SBS}}\right)$ como se indica en la Ec. 11.

$$
P_{S B S}=N_{S l} \cdot N_{a} \cdot P_{a}
$$

\subsection{CAPEX y OPEX en el EPC 9 irtualizado}

Si se profundiza un poco más y se aprovechan aún más los beneficios que proporcionan la tecnología NFV, se pueden virtualizar los elementos principales del núcleo de la red 5G (EPC): MME (Mobility Management Entity), HSS (Home Subscriber Server), SGW (Serving Gateway) y PGW (Packet Data Network Gateway).

Siendo $\mathrm{N}_{\text {SERV }}$ el número total de servidores y $\mathrm{C}_{\text {SERV }}$ el coste de un servidor, se puede definir el coste total de la adquisición de los servidores que son necesarios, tal y como muestra la Ec. 12.

$$
C_{S E R V-\text { total }}=N_{S E R V} \cdot C_{S E R V}
$$

Por lo tanto, se puede definir el CAPEX total para virtualizar el EPC (CAPEX $\mathrm{VEPC}_{\mathrm{V}}$ ), tal y como se muestra en la Ec. 13, teniendo en cuenta el coste total de los servidores requeridos para la virtualización $\left(\mathrm{C}_{\text {SERV-total }}\right)$, los costes de licencia software $\left(\mathrm{C}_{\mathrm{LIC}}\right)$ y los costes asociados al despliegue e instalación inicial $\left(\mathrm{C}_{\mathrm{INI}}\right)$.

$$
\text { CAPEX } X_{v E P C}=C_{S E R V-t o t a l}+C_{L I C}+C_{I N I}
$$

Por otro lado, para definir el OPEX del EPC virtualizado se tendrá en cuenta el consumo energético de todos los servidores necesarios para alojar el número requerido de máquinas virtuales. Para virtualizar cualquier elemento del EPC ( $\left.\mathrm{E}_{\mathrm{EPC}}\right)$, el número de máquinas virtuales necesarias para dicha demanda se determinará mediante el requisito máximo de CPU, memoria, red o almacenamiento: Ec. 14.

$$
V M S_{E_{E P C}}=\operatorname{máx}\left(V M_{C P U-E_{E P C}}, V M_{R A M-E_{E P C}}, V M_{A L M-E_{E P C}}, V M_{R E D-E_{E P C}}\right)
$$

Los requisitos de CPU, memoria RAM, almacenamiento y red son definidos tal y como se muestran en las Ec. $15-18$, respectivamente.

$$
\begin{aligned}
V M_{C P U-E_{E P C}} & =\left(\frac{C P U_{E_{E P C}}}{C P U_{\text {porV } M}}\right) \\
V M_{R A M-E_{E P C}} & =\left(\frac{R A M_{E_{E P C}}}{R A M_{\text {porVM }}}\right) \\
V M_{A L M-E_{E P C}} & =\left(\frac{A L M_{E_{E P C}}}{A L M_{\text {porVM }}}\right) \\
V M_{R E D-E_{E P C}} & =\left(\frac{R E D_{E_{E P C}}}{R E D_{\text {porVM }}}\right)
\end{aligned}
$$

El número de servidores requeridos para albergar cada elemento del EPC viene dado por la Ec. 19.

$$
N_{S E R V-E_{E P C}}=\frac{V M S_{E_{E P C}}}{V M S_{\text {porSERV }}}
$$

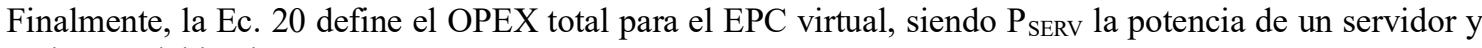
$\mathrm{C}_{\mathrm{kWh}}$ el coste del $\mathrm{kWh}$. 


$$
O P E X_{v E P C}=\left[\sum_{i \in E_{E P C}} N_{S E R V_{i}}\right] \cdot P_{S E R V} \cdot C_{k W h}
$$

\section{ESTUDIO REAL SOBRE LA RCT: RESULTADOS NUMÉRICOS}

Extremadura cuenta con una infraestructura de fibra óptica a la que la Junta de Extremadura denominó RCT, cuyo principal objetivo es proporcionar servicios de comunicaciones avanzadas a los centros tecnológicos ubicados en la región. Dada la gran capacidad de transmisión que ofrece la RCT, se exceden las necesidades de los centros conectados inicialmente. Por ello, se puede reutilizar el sobredimensionamiento de red para la prestación de servicios de acceso a las infraestructuras de telecomunicaciones.

Por lo tanto, en esta sección se evaluará la implantación de mecanismos de nueva generación en la RCT, con el objetivo de cuantificar los costes operacionales y de capital. Los parámetros utilizados en el análisis (S.F. Yunas et al. 2014) (LTE Multi-Plataform Base Station) (N. Zhang and H. Hämmäinen 2015) por defecto son: $\mathrm{C}_{\mathrm{dBS}}=\mathrm{C}_{\mathrm{dSBS}}=5.000 € ; \mathrm{C}_{\mathrm{BS}}=15.596 € ; \mathrm{P}_{\mathrm{a}}=615 \mathrm{~W} ; \mathrm{N}_{\mathrm{a}}=4 ; \mathrm{C}_{\mathrm{kWh}}=0,14 € ; \mathrm{C}_{\mathrm{OAM}}=4.000 € / \mathrm{año} ; \mathrm{C}_{\mathrm{ALQ}}=$ $1000 € / \mathrm{año} ; \mathrm{R}_{\max }=2 \mathrm{~km} ; \mathrm{C}_{\mathrm{LIC}}=5.000 € ; \mathrm{C}_{\mathrm{INI}}=30.000 € ; \mathrm{C}_{\mathrm{SERV}}=5.500 € ; \mathrm{P}_{\mathrm{SERV}}=400 \mathrm{~W} ; \mathrm{CPU}_{\mathrm{MME}}=\mathrm{CPU}_{\mathrm{HSS}}$ $=\mathrm{CPU}_{\mathrm{SGW}}=\mathrm{CPU}_{\mathrm{PGW}}=6$ cores; $\mathrm{RAM}_{\mathrm{MME}}=\mathrm{RAM}_{\mathrm{HSS}}=\mathrm{RAM}_{\mathrm{SGW}}=\mathrm{RAM}_{\mathrm{PGW}}=40 \mathrm{~GB} ; \mathrm{ALM}_{\mathrm{MME}}=\mathrm{ALM}_{\mathrm{HSS}}$ $=\mathrm{ALM}_{\mathrm{SGW}}=\mathrm{ALM}_{\mathrm{PGW}}=1 \mathrm{~TB} ; \mathrm{RED}_{\mathrm{MME}}=\mathrm{RED}_{\mathrm{HSS}}=\mathrm{RED}_{\mathrm{SGW}}=\mathrm{RED}_{\mathrm{PGW}}=30 \mathrm{Gbps} ; \mathrm{CPU}_{\mathrm{porVM}}=2$ cores; $\mathrm{RAM}_{\text {porVM }}=8 \mathrm{~GB} ; \mathrm{ALM}_{\text {porVM }}=150 \mathrm{~GB} ; \mathrm{RED}_{\text {porVM }}=40 \mathrm{Gbps} ; \mathrm{VMS}_{\text {porSERV }}=10$.

En primer lugar, se han calculado los costes de capital y operación en relación al número de estaciones base desplegadas. La Fig. 1 muestra la comparativa entre las dos arquitecturas desplegadas (RAN y C-RAN).

En ambos casos, el CAPEX aumenta de manera lineal con respecto al número de estaciones base desplegadas. Se pueden conseguir ahorros de los costes de capital del orden del 70\% cuando se despliegan 100 BS bajo una arquitectura C-RAN, con respecto a una arquitectura RAN tradicional. En el caso del OPEX, al ser costes relacionados con el mantenimiento de la infraestructura, en este artículo se han considerado los costes asociados al primer año de operación, es decir, OPEX anual. En el caso de C-RAN se consiguen ahorros de OPEX de aproximadamente un $60 \%$ con respecto a la arquitectura RAN.

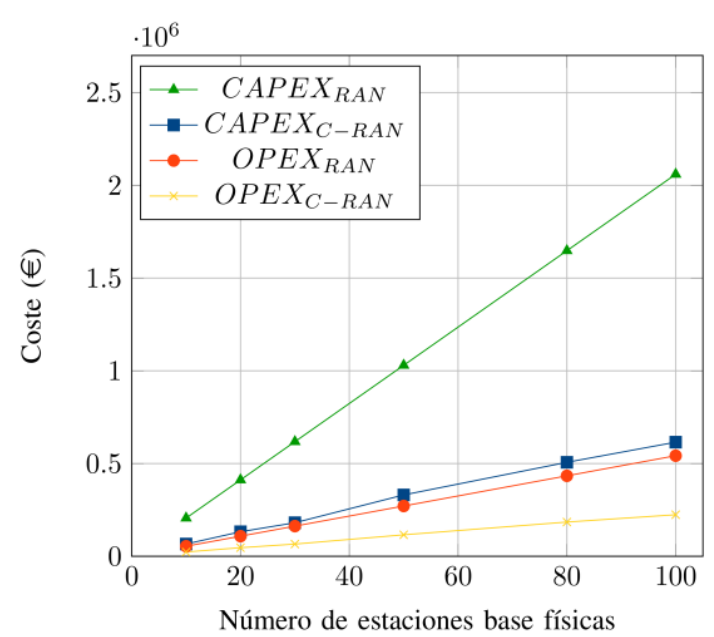

Figura 1. Análisis de CAPEX y OPEX: RAN vs C-RAN

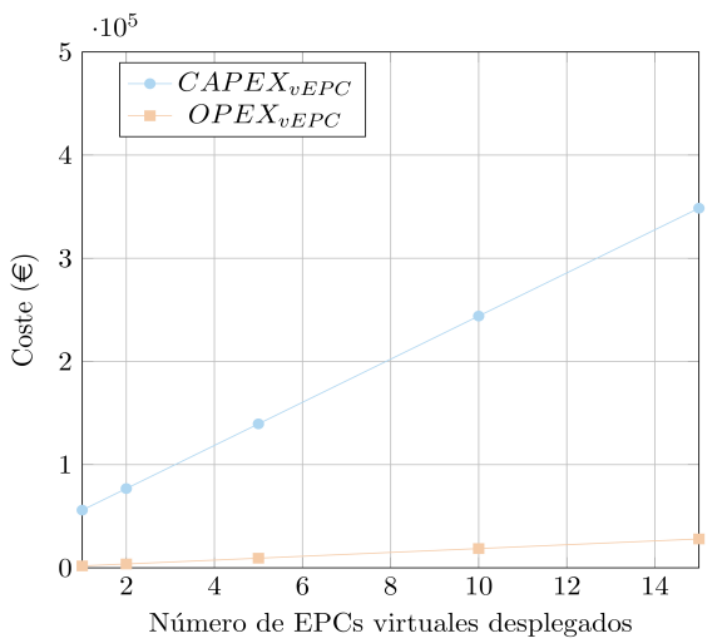

Figura 2. Análisis de CAPEX y OPEX del vEPC

FiAdemás, se han calculado los costes de capital y operación en relación al número de EPCs virtuales desplegados. La Figura 2 muestra la evolución de los costes que aumentan de manera lineal con respecto al número de vEPCs desplegados. 


\section{CONCLUSIONES}

Debido al rápido crecimiento del tráfico de datos móviles y los nuevos servicios demandados por los usuarios, los proveedores de servicios de telecomunicaciones han propuesto nuevas formas de prestación de servicios en términos de flexibilidad, agilidad y ahorro de costes a través de la gestión y ahorro de los costes de operación y los costes de capital.

En este trabajo se propone un enfoque para reducir el impacto de NFV y SDN en el coste total de despliegue y mantenimiento para un operador de red. Se presenta un modelo de análisis de costes que proporciona una visión técnica y económica para decidir qué arquitectura es la más acertada a la hora de desplegar las tecnologías de red y los servicios que han de ser desarrollados. Además, se ha llevado a cabo un análisis del despliegue del EPC virtualizado sobre la RCT. El análisis realizado revela que la arquitectura propuesta proporciona importantes ahorros de CAPEX y OPEX. Los resultados experimentales obtenidos de la comparación entre el caso tradicional y el caso virtualizado muestran que el CAPEX para la red de acceso virtualizada se puede reducir hasta un $70 \%$ con respecto al caso tradicional. En comparación con la red de acceso tradicional, el OPEX para la red de acceso virtualizada puede reducirse hasta un $59 \%$.

\section{AGRADECIMIENTO}

Este trabajo está financiado en parte por el Fondo Europeo de Desarrollo Regional (FEDER) Programa Operativo 2014-2020 de Extremadura a través del proyecto CultivData (2018.14.02.332A.444.00), y, en parte, a través de los Fondos Europeos de Desarrollo Regional bajo el proyecto IB18003.

\section{REFERENCIAS}

B. Blanco et al. 2017. Technology pillars in the architecture of future 5G mobile networks: NFV, MEC and SDN. In Computer Standards and Interfaces, vol. 54, pp. 216-228.

B. Han et al. 2015. Network function virtualization: Challenges and opportunities for innovations. In IEEE Communications Magazine, vol. 53, no. 2, pp. 90-97.

B. Naudts et al. 2016. How can a mobile service provider reduce costs with software-defined networking? In International Journal of Network Management, vol. 26, no. 1, pp. 56-72.

B.A.A.Nunes et al. 2014. A Survey of Software-Defined Networking: Past, Present, and Future of Programmable Networks. In IEEE Communications Surveys \& Tutorials, vol. 16, no. 3, pp. 1617-1634.

Cisco Systems Inc, 2019. Cisco Visual Networking Index: Forecast and Trends, 2017-2022. White Paper.

D. Pompili et al. 2015. Dynamic provisioning and allocation in Cloud Radio Access Networks (C-RANs). In Ad Hoc Networks, vol. 30, pp. 128-143.

E. Hernández et al. 2015. How will nfv/sdn transform service provider opex?. In IEEE Network, vol. 29, no. 3.

I.-F. Akyildiz et al. 2015. SoftAir: A software defined networking architecture for $5 G$ wireless systems. In Computer Networks, vol. 85, pp. 1-18.

J. A. Cabrera et al. 2019. Fitzek. Softwarization and Network Coding in the Mobile Edge Cloud for the Tactile Internet. In Proceedings of the IEEE, vol. 107, no. 2, pp. 350-363.

LTE Multi-Platform Base Station. https://www.winncom.com/en/ products/998- 03- 536

N. Zhang and H. Hämmäinen 2015. Cost Efficiency of SDN in LTE-based Mobile Networks: Case Finland. In International Conference and Workshops on Networked Systems.

R. Mijumbi et al. 2016. Network Function Virtualization: State-of-the-Art and Research Challenges. In IEEE Communications Surveys Tutorials, vol. 18, pp. 236-262.

S. Verbrugge et al. 2006. Methodology and input availability parameters for calculating OpEx and CapEx costs for realistic network scenarios. In Journal of Optical Networking, vol. 5, no. 6, pp. 509-520.

S.F. Yunas et al. 2004. Relation between base station characteristics and cost structure in cellular systems. In IEEE Personal, Indoor and Mobile Radio Communications, vol. 4, pp. 2627-2631, 2004.

S.F. Yunas et al. 2014. Techno-economical analysis and comparison of legacy and ultra-dense small cell networks. In Local Computer Networks. 